\title{
ANTIDIABETIC MEDICATION PERSISTENCE AND IMPLEMENTATION IN PATIENTS WITH CHRONIC KIDNEY DISEASE
}

CHU de Québec Université Laval Centre de recherche

\section{Viet Thanh Truong ${ }^{1,2}$, Jocelyne Moisan ${ }^{1,2}$, Edeltraut Kröger', Serge Langlois ${ }^{3}$, Jean-Pierre Grégoire ${ }^{1,2}$}

' Faculty of Pharmacy, Université Laval, Québec, QC, Canada.

2Population Health and Optimal Health Practices Research Unit, CHU de Québec -

Université Laval Research Centre, Québec, QC, Canada. FFaculty of Medicine, Université Laval, Québec, QC, Canada.

\section{INTRODUCTION}

Glycemic control contributes to both slowing chronic kidney disease (CKD) progression and reducing the risk of cardiovascular mortality and morbidity.

Most diabetic patients have to use antidiabetic drugs (AD) to achieve gly cemic control.

- Those drugs have to be taken every day during a long period of time.

\section{AIMS}

Among individuals suffering from CKD newly treated with an $A D$ :

. To estimate persistence with $A D 1$ year after $A D$ treatment initiation.

2. Among those persisting, to estimate treatment implementation in the year following $A D$ initiation.

3. To identify factors associated with persistence.

4. To identify factors associated with implementation.

\section{METHODS}

Study design and data sources

We carried out a cohort study using Quebec administrative databases. Databases provide information on patient's demographics (age, sex, socioeconomic status), outpatient medical services, date of diagnosis, physician specialty, use of drugs and hospitalizations.

Study population

- Inclusion criteria:

Individuals aged $\geq 18$ years old newly diagnosed with CKD between 1 January 2000 and 31 December 2011

Had a first claim for any AD at the time of CKD diagnosis or after.

- Exclusion criteria:

Individuals not eligible to the RAMQ drug plan 365 days before and 365 days after the date of $A D$ treatment initiation.

Those who moved out from the province of Quebec.

Those who died on the date of their $1^{\text {st }} \mathrm{CKD}$ diagnosis or died during the hospital stay in which CKD was first diagnosed.

Variables

Outcome variables

- Persistence with AD

Individuals had a provision of any $A D$ at the 1 st year anniversary date of treatment initiation according to most

- Implementation of AD

If individuals were persistent and had $\geq 80 \%$ of days covered by any AD between CKD diagnosis and day 365

Figure 1. Timeline measure of variable

CKD

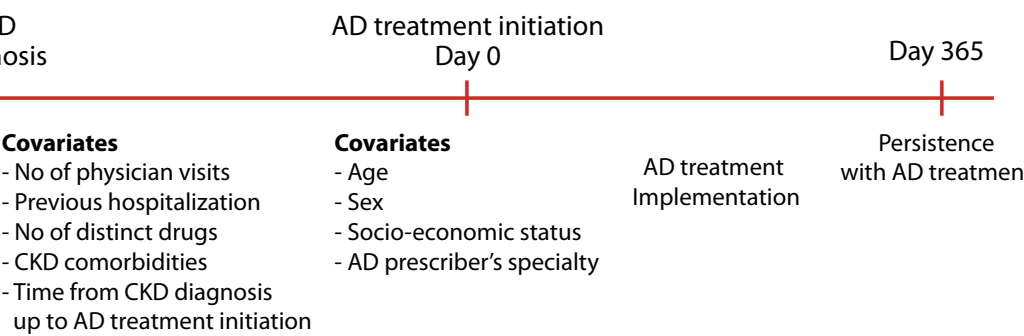

tatistical analysis

We identified factors associated with persistence and with implementation using modified Poisson regression.

- Sensitivity analyses were conducted for persistence (permissible gaps of 1.0 and 1.5 time the days' supply) and implementation $(\geq 70 \%, \geq 80$ and $\geq 90 \%$ of days covered).

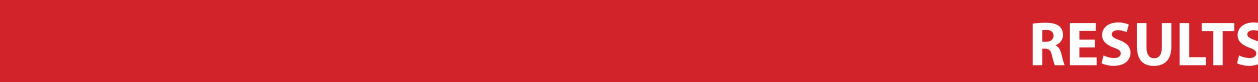

Among 6,671 individuals included in our study population, 5,128 (76.9\%) were persistent.

Table 1. Adjusted prevalence ratios of patients' characteristics and persistence with antidiabetic drug treatment 365 days after treatment initiation $(\mathrm{N}=6,671)$

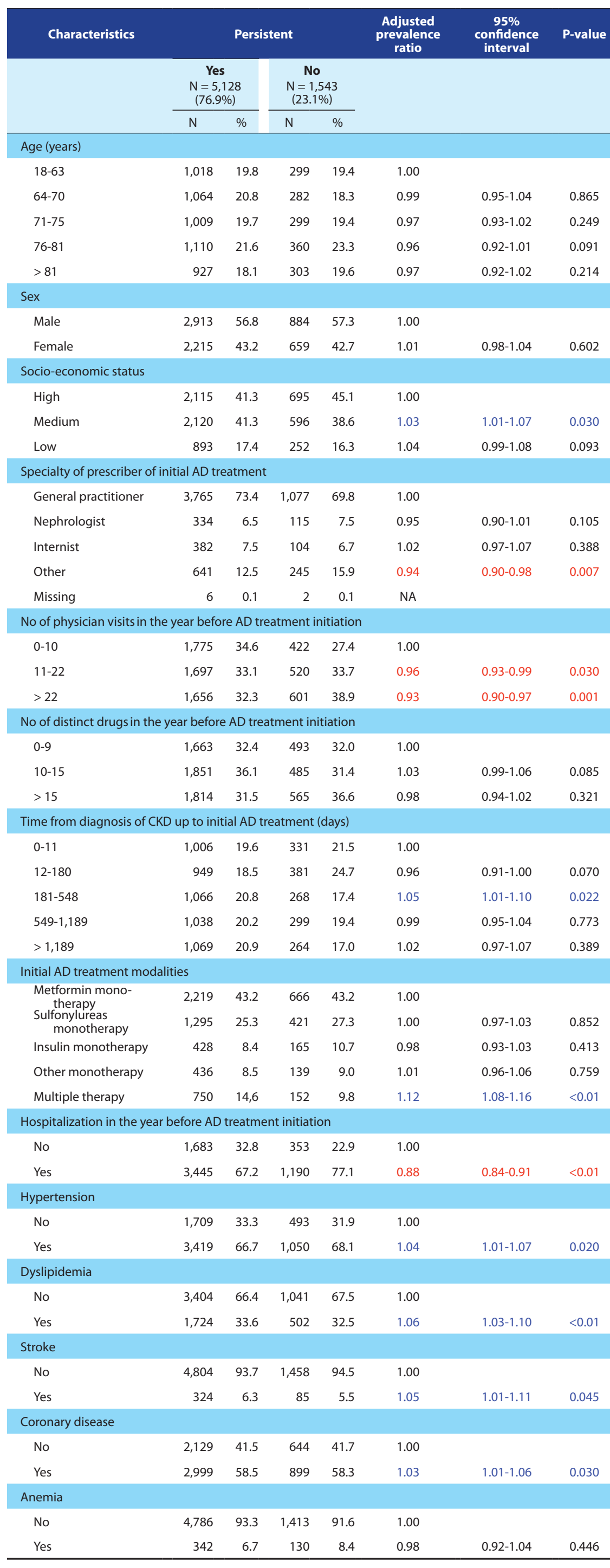

Table 2. Adjusted prevalence ratios of patients' characteristics and implementation of antidiabetic drug treatment among those persisting with heir antidiabetic treatment 365 days after treatment initiation $(\mathrm{N}=5,128)$

\begin{tabular}{|c|c|c|c|c|c|c|c|}
\hline \multirow[t]{3}{*}{ Characteristics } & \multicolumn{4}{|c|}{ Adequate implementation } & \multirow[t]{3}{*}{$\begin{array}{l}\text { Adjusted } \\
\text { valence ratio }\end{array}$} & \multirow[t]{3}{*}{$\begin{array}{c}95 \% \\
\text { confdence } \\
\text { interval }\end{array}$} & \multirow[t]{3}{*}{ P-value } \\
\hline & \multicolumn{2}{|c|}{$\begin{array}{c}\text { Yes } \\
\mathrm{N}=4.506 \\
(87.906)\end{array}$} & \multicolumn{2}{|c|}{$\begin{array}{c}\text { No } \\
N=622 \\
(12.120)\end{array}$} & & & \\
\hline & $\mathrm{N}$ & $\%$ & $\mathrm{~N}$ & $\%$ & & & \\
\hline \multicolumn{8}{|l|}{ Age (years) } \\
\hline $18-63$ & 875 & 19.4 & 143 & 23.0 & 1.00 & & \\
\hline $64-70$ & 933 & 20.7 & 131 & 21.1 & 1.03 & $0.99-1.06$ & 0.083 \\
\hline 71-75 & 868 & 19.3 & 141 & 22.7 & 1.01 & $0.97-1.05$ & 0.617 \\
\hline $76-81$ & 983 & 21.8 & 127 & 20.4 & 1.03 & $0.99-1.07$ & 0.095 \\
\hline$>81$ & 847 & 18.8 & 80 & 12.8 & 1.06 & $1.02-1.10$ & 0.002 \\
\hline \multicolumn{8}{|l|}{ Sex } \\
\hline Male & 2,518 & 55.9 & 395 & 63.5 & 1.00 & & \\
\hline Female & 1,988 & 44.1 & 227 & 36.5 & 1.02 & $1.01-1.04$ & 0.048 \\
\hline \multicolumn{8}{|l|}{ Socio-economic status } \\
\hline High & 1,827 & 40.5 & 288 & 46.3 & 1.00 & & \\
\hline Medium & 1,882 & 41.8 & 238 & 38.3 & 1.01 & $0.98-1.03$ & 0.463 \\
\hline Low & 797 & 17.7 & 96 & 15.4 & 1.04 & $1.01-1.07$ & 0.029 \\
\hline \multicolumn{8}{|c|}{ Specialty of prescriber of initial AD treatment } \\
\hline General practitioner & 3,339 & 74.1 & 426 & 68.5 & 1.00 & & \\
\hline Nephrologist & 283 & 6.3 & 51 & 8.2 & 0.97 & $0.92-1.01$ & 0.156 \\
\hline Internist & 333 & 7.4 & 49 & 7.9 & 0.98 & $0.94-1.02$ & 0.335 \\
\hline Other & 548 & 12.1 & 93 & 14.9 & 0.96 & $0.93-0.99$ & 0.046 \\
\hline Missing & 3 & 0.1 & 3 & 0.5 & NA & & \\
\hline \multicolumn{8}{|c|}{ No of physician visits in the year before $A D$ treatment initiation } \\
\hline $0-10$ & 1,532 & 34.0 & 243 & 39.1 & 1.00 & & \\
\hline $11-22$ & 1,528 & 33.9 & 169 & 27.2 & 1.03 & $1.01-1.06$ & 0.020 \\
\hline$>22$ & 1,446 & 32.1 & 210 & 33.7 & 0.99 & $0.96-1.02$ & 0.675 \\
\hline \multicolumn{8}{|c|}{ No of distinct drugs in the year before $A D$ treatment initiation } \\
\hline $0-9$ & 1,409 & 31.3 & 254 & 40.8 & 1.00 & & \\
\hline $10-15$ & 1,651 & 36.6 & 200 & 32.2 & 1.05 & $1.02-1.07$ & 0.001 \\
\hline$>15$ & 1,446 & 32.1 & 168 & 27.0 & 1.05 & $1.02-1.08$ & 0.001 \\
\hline \multicolumn{8}{|c|}{ Time from diagnosis of CKD up to initial AD treatment (days) } \\
\hline $0-11$ & 891 & 19.8 & 115 & 18.5 & 1.00 & & \\
\hline 12-180 & 838 & 18.6 & 111 & 17.9 & 0.99 & $0.97-1.03$ & 0.982 \\
\hline $181-548$ & 935 & 20.8 & 131 & 21.1 & 0.98 & $0.96-1.02$ & 0.515 \\
\hline $549-1,189$ & 899 & 19.9 & 139 & 22.3 & 0.99 & $0.96-1.03$ & 0.575 \\
\hline$>1,189$ & 943 & 20.9 & 126 & 20.2 & 1.01 & $0.98-1.05$ & 0.418 \\
\hline \multicolumn{8}{|c|}{ Initial AD treatment modalities } \\
\hline $\begin{array}{l}\text { Metformin mono- } \\
\text { theraov }\end{array}$ & 1,939 & 43.0 & 280 & 45.0 & 1.00 & & \\
\hline $\begin{array}{l}\text { Sulfonylureas } \\
\text { monothera }\end{array}$ & 1,130 & 25.1 & 165 & 26.5 & 0.99 & $0.97-1.02$ & 0.798 \\
\hline Insulin monotherapy & 366 & 8.1 & 62 & 10.0 & 0.98 & $0.95-1.03$ & 0.592 \\
\hline Other monotherapy & 375 & 8.3 & 61 & 9.8 & 0.98 & $0.94-1.02$ & 0.420 \\
\hline Multiple therapy & 696 & 15.5 & 54 & 8.7 & 1.06 & $1.03-1.09$ & $<0.01$ \\
\hline Hospitalization in the ye & efore $A L$ & treatm & nt initic & & & & \\
\hline No & 1,449 & 32.2 & 234 & 37.6 & 1.00 & & \\
\hline Yes & 3,057 & 67.8 & 388 & 62.4 & 1.01 & $0.97-1.04$ & 0.855 \\
\hline Hypertension & & & & & & & \\
\hline No & 1,485 & 33.0 & 224 & 36.0 & 1.00 & & \\
\hline Yes & 3,021 & 67.0 & 398 & 74.0 & 1.01 & $0.98-1.03$ & 0.901 \\
\hline Dyslipidemia & & & & & & & \\
\hline No & 2,967 & 65.9 & 437 & 70.3 & 1.00 & & \\
\hline Yes & 1,539 & 34.1 & 185 & 29.7 & 1.02 & $0.99-1.04$ & 0.163 \\
\hline Stroke & & & & & & & \\
\hline No & 4,216 & 93.6 & 588 & 94.5 & 1.00 & & \\
\hline Yes & 290 & 6.4 & 34 & 5.5 & 1.02 & $0.98-1.06$ & 0.465 \\
\hline Coronary disease & & & & & & & \\
\hline No & 1,851 & 41.1 & 278 & 44.7 & 1.00 & & \\
\hline Yes & 2,655 & 58.9 & 344 & 55.3 & 1.01 & $0.98-1.03$ & 0.797 \\
\hline Anemia & & & & & & & \\
\hline No & 4,198 & 93.2 & 588 & 94.5 & 1.00 & & \\
\hline Yes & 308 & 6.8 & 34 & 5.5 & 1.01 & $0.97-1.05$ & 0.612 \\
\hline
\end{tabular}

\section{CONCLUSION}

Results could help target individuals who need help to better manage their $\mathrm{AD}$ treatment.
Overall, 32\% of patients with CKD who initiated an AD may not fully benefit from it as they were either non-persistent or had inadequate treatmen implementation

11 characteristics were associated with persistence and 8 with implemen tation. Only 3 were associated with both (another specialist as prescriber of the first AD claim, between 11 and 22 physician visits in the year befor AD treatment initiation, and a multiple therapy at treatment initiation). This confirms that persistence and implementation are two different constructs of adherence.

\section{Strengths}

- Large sample size, new-user design, population-based for those aged 65 years.

- Real-life practice setting

Assessment of both persistence and implementation, two different constructs of adherence.

Limitations

Drugs claimed were assumed to be taken.

- Lack of information on psychosocial variables (e.g. patient's perception of risk of disease and benefits of the treatment) that are likely to influence drug persistence and implementation. 